\title{
Uso de biofertilizantes en el desarrollo vegetativo y productivo de plantas de camu-camu en Ucayali, Perú ${ }^{1}$
}

\author{
Carlos Abanto-Rodríguez 2,6*(1), Gerson Manuel Soregui Mori ${ }^{3}$, Mario Herman Pinedo Panduro ${ }^{4}$, \\ Ena Vilma Velazco Castro ${ }^{3}$, Elvis Javier Paredes Dávila ${ }^{4}$ Eduardo Medeiros de Oliveira ${ }^{5}$
}

$10.1590 / 0034-737 X 201966020005$

\begin{abstract}
RESUMEN
Para aumentar la calidad y el rendimiento de frutos en plantaciones de camu-camu, actualmente se viene buscando alternativas de producción orgánica. En ese sentido, el presente trabajo tuvo por objetivo determinar el efecto de diferentes biofertilizantes en el desarrollo vegetativo y productivo de plantas de camu-camu. Para ello, fue conducido un experimento mediante un diseño de bloques completamente al azar (DBCA) en esquema factorial (5Bx5C), con cuatro repeticiones y una planta por unida experimental. El primer factor (B) estuvo constituido por cinco tipos de biofertilizantes: B1) vacaza (estiércol de vaca), B2) gallinaza (estiércol de gallina de postura), B3) guano de isla (estiércol de aves de mar), B4) pollinaza (estiércol de pollo) y B5) sedimentos de rio, y el segundo factor (C) por cinco concentraciones de biofertilizante: C1) 0,C2) 2, C3) 4,C4) 6 y C5) 8\%. Después de 210 días, fue verificado que, el biofertilizante guano de isla estimuló mayor emisión de brotes nuevos $(1773,73)$ y el biofertilizante vacaza presentó los mejores resultados de botones florales, número de frutos de cosecha y rendimiento de frutos $\left(\mathrm{t} \mathrm{ha} \mathrm{a}^{-1}\right.$ ) con 4611,67; 2926,85 y 28,8 en las dosis 6; 8 y $8 \%$, respectivamente. Se concluye que, el biofertilizante vacaza (VA) incrementó la calidad y el rendimiento de frutos de camu-camu en suelos de restinga. Así también, el biofertilizante guano de isla (GI) fue el segundo en presentar los mejores resultados, sin embargo, el alto contenido de $\mathrm{N}$ retardó el desarrollo normal de las fases fenológicas.
\end{abstract}

Palabras-clave: Myrciaria dubia (Kunth) McVaugh; compuestos orgánicos; manejo agronómico; fertilización orgánica; restinga.

\section{RESUMO}

\section{Uso de biofertilizante no desenvolvimento vegetativo e produtivo de plantas de camu-camu em Ucayali, Peru}

Para aumentar a qualidade e o rendimento de fruto em pomares de camu-camu, atualmente procuram-se alternativas de produção orgânica. Nesse sentido, objetivou-se determinar o efeito de diferentes tipos de biofertilizantes no desenvolvimento vegetativo e produtivo de plantas de camu-camu. Para isso, foi conduzido um experimento em delineamento em blocos ao acaso (DBC) em esquema fatorial 5B x 5C com quatro repetições e uma planta por unidade experimental. $\mathrm{O}$ primeiro fator (B) foi constituído por cinco tipos de biofertilizante: B1) esterco de vaca, B2) esterco de galinha de postura, B3) guano (esterco de aves de mar), B4) esterco de frango e B5) sedimentos de rio, e o segundo fator (C) por cinco concentrações de biofertilizante: C1) 0, C2) 2, C3) 4,C4) 6 y C5) 8\%. Após 210 dias foi verificado que o guano estimulou a maior emissão de brotos novos $(1773,73)$ e o esterco de vaca apresentou os melhores resultados de número de botões florais, frutos na colheita e rendimento de fruto $\left(\mathrm{t} \mathrm{ha}^{-1}\right)$ com 4611,67; 2926,85 e 28,8, nas concentrações de 6; 8 e $8 \%$,

Submetido em 04/05/2018 e aprobado 12/04/2019

${ }^{1}$ Trabajo de investigación financiado por el Programa Nacional de Innovación para la Competitividad y Productividad (INNOVATE-PERU), de acuerdo al Convenio N ${ }^{a}$ 403-PNICPPIAP-2014-IIAP.

${ }^{2}$ Instituto de Investigaciones de la Amazonía Peruana-IIAP, Programa de Investigación "PROBOSQUES", Yarinacocha, Ucayali, Perú;

${ }^{3}$ Universidad Nacional Intercultural de la Amazonía-UNIA, Departamento Académico de Ingeniería Agroforestal Acuícola, San José, Yarinacocha, Perú. m.soregui01@ gmail.com; evelazcoc@unia.edu.pe

${ }^{4}$ Instituto de Investigaciones de la Amazonía Peruana-IIAP, Programa de Investigación PROBOSQUES, Iquitos, Loreto, Perú. mpinedo@iiap.org.pe; eparedes@iiap.org.pe

${ }_{5}^{5}$ Universidade Federal de Roraima, Programa de Pós-graduação em Agronomia-POSAGRO, Boa Vista, Roraima, Brasil. eduardo-medeiros@outlook.com

Universidade Federal de Roraima, Programa de

${ }^{6}$ Pós-graduação em Biodiversidade e Biotecnologia da Amazônia Legal (REDE BIONORTE), Boa Vista, Roraima, Brasil. carforestal24@gmail.com 
respectivamente. Conclui-se que o esterco de vaca acompanhado de tratos culturais incrementou a qualidade e o rendimento de fruto de camu-camu em solos de várzea. Além disso, o guano de ilha foi o segundo em apresentar os melhores resultados, porém o alto conteúdo de nitrogênio retardou o desenvolvimento normal das fases fenológicas.

Palavras-chave: Myrciaria dubia (Kunth) McVaugh; compostos orgânicos; manejo agronômico; adubação orgânica; várzea.

\section{INTRODUCCIÓN}

Científicamente está demostrado que los seres humanos no son capaces de sintetizar vitamina $\mathrm{C}$ en el organismo, por lo tanto, es necesario que sea injerida de fuentes externas (Chatterjee et al., 1975; Naidu, 2003). En ese sentido, hombres y mujeres de todo mundo, están buscando nuevos productos naturales que posean altas concentraciones de vitamina C. Uno de estos productos es el camu-camu, el cual se destaca por ser una fuente importante de antioxidantes, debido a su alta concentración de ácido ascórbico (AA), conteniendo hasta 3079,0 mg de AA $100 \mathrm{~g}^{-1}$ de pulpa (Pinedo et al., 2010; Abanto et al., 2014). Además, posee otros compuestos antioxidantes como carotenoides, antocianinas (Neves et al., 2015), y es una importante fuente de potasio, hierro, calcio, fósforo y varios tipos de aminoácidos como la serina, valina y leucina (Alves et al., 2002).

Debido a sus características nutricionales, el cultivo está en plena expansión en los países de Perú, Brasil y Bolivia. En relación a Perú, actualmente es el mayor productor con una producción aproximada de 10615,0 t de fruto año-1 (DRAL, 2015; DRSAU, 2017). Además, es el primer exportador mundial, siendo que los principales compradores son: Japón, Estados Unidos y Reino Unido, con participación en el mercado de 41, 97; 31,78 y 4,83\%, respectivamente y a su vez, los productos más exportados son pulpa, polvo y cápsulas (SUNAT, 2018).

Por todo esto, el cultivo de camu-camu en la actualidad es considerado como la primera especie nativa de importancia económica y ecológica que se desarrolla en las riberas de los ecosistemas inundables de la Amazonía Peruana (Pinedo et al., 2010). Las condiciones de producción en estos ambientes han sido propicias para que camu-camu sea conocido y comercializado a nivel nacional e internacional como un producto orgánico, dado que las prácticas de fertilización mineral, muchas veces, es opcional debido a que las plantas año tras año son nutridas naturalmente con los sedimentos que son transportados durante el periodo de creciente de los ríos.

Sin embargo, en los últimos años el rendimiento de frutos ha sido afectado considerablemente llegando a una producción aproximada de 1,5 a 2,5 t ha ${ }^{-1}$ (DRSAU, 2017). Esta disminución está siendo afectada según Abanto et al. (2016), principalmente por reducción de algunos elementos esenciales como N, K y Mg en los suelos, que es causado básicamente por la variación de los niveles de inundación en las diferentes zonas de cultivo y la falta de tecnologías de fertilización de bajo costo para reponer los nutrientes que son extraídos año tras año en las cosechas. Otro factor que ha contribuido para la disminución de la producción de fruto es el aumento de plagas y enfermedades debido a prácticas inadecuadas de manejo agronómico.

En tal sentido para recuperar y elevar los niveles de producción, urge desarrollar tecnologías. Una de estas tecnologías es el uso de técnicas de fertilización orgánica que consiste en la incorporación y/o aplicación al suelo y a las plantas compuestos orgánicos elaborados a partir de residuos de origen vegetal y animal en diferentes fases de descomposición, humificación y mineralización (Souza \& Alcântara, 2007). Dentro de los compuestos orgánicos, el biofertilizante se destaca por ser líquido y por aportar nutrientes esenciales para las plantas. El biofertilizante puede ser utilizado de diversas maneras, siendo que, el método más eficiente es la aplicación de pulverizaciones foliares, porque promueve un efecto más rápido (Medeiros \& Lopes, 2006).

Según, Vairo dos Santos (1992), el producto puede ser aplicado en concentraciones que varían de 5\% a 20\%, dependiendo del efecto esperado para cada cultivo, pues el efecto nutricional es obtenido a partir de las concentraciones más bajas (5\% a $10 \%$ ) y el efecto insecticida, fungicida y bactericida en concentraciones más elevadas (10\% a 20\%).

En ese contexto, verificado la importancia y efectividad de los biofertilizantes en el manejo agronómico de los cultivos, este trabajo tuvo como objetivo determinar el efecto de diferentes tipos de biofertilizantes en el desarrollo vegetativo y productivo de plantas de camu-camu en Ucayali, Perú.

\section{MATERIAL Y MÉTODOS}

La primera etapa de la investigación se realizó en el Centro de Investigaciones Dale E. Bandy del Instituto de Investigaciones de la Amazonía Peruana - IIAP, ubicado en el km 12,4 de la Carretera Federico Basadre, distrito de 
Yarinacocha, provincia de Coronel Portillo, Región Ucayali, situado a $8^{\circ} 23^{\prime}$ 52,60" de latitud Sur y $74^{\circ} 38^{\prime} 24,95^{\prime}$ ' de longitud Oeste y a una altitud de $154 \mathrm{msnm}$ y la segunda etapa fue realizada en la parcela experimental ubicada en el caserío Pucallpillo de Manantay ubicada en las coordenadas geográficas $8^{\circ} 27^{\prime} 42^{\prime \prime}$ de longitud Sur y $74^{\circ} 30^{\prime} 84^{\prime \prime}$ de longitud Oeste, a una altitud de $145 \mathrm{msnm}$. Correspondiente a la planicie aluvial de terraza baja inundable.

Los biofertilizantes fueron elaborados en el área experimental "camu-camu" del IIAP-Ucayali, en un ambiente techado con la finalidad de protegerlos del sol y lluvia, los insumos para la elaboración fueron obtenidos de diferentes puntos de la ciudad de Pucallpa. Los biofertilizantes elaborados fueron: B1) vacaza (estiércol de vaca), B2) gallinaza (estiércol de gallina de postura), B3) guano de isla (estiércol de aves de mar), B4) pollinaza (estiércol de pollo) y B5) sedimento de rio.

Los cinco tipos de biofertilizantes fueron producidos en cilindros de $200 \mathrm{~L}$; inicialmente fue adicionado 50 litros de agua y $50 \mathrm{~kg}$ de estiércol, posteriormente fue agitado hasta conseguir una mezcla homogénea y finalmente fue agregado $1 \mathrm{~kg}$ de chancaca (producto orgánico obtenido de la evaporación del jugo de la caña de azúcar) con la finalidad de inyectar energía necesaria para activar el metabolismo y conseguir fermentación de manera más rápida.

La mezcla permaneció en cada uno de los cilindros durante un periodo de 90 días (junio - agosto, 2015), siendo que estos fueron agitados todos los días con una tablilla de madera para incrementar la proliferación de microorganismos y para evitar la sedimentación de los diferentes insumos en los cilindros. Después de este periodo, los biofertilizantes fueron tamizados y filtrados con la finalidad de tener un producto libre de impurezas para no obstruir la bomba aspersora al momento de la aplicación. Seguidamente fueron colectadas muestras de $500 \mathrm{~mL}$ de cada uno de los biofertilizantes y en seguida fueron llevadas al laboratorio de análisis de suelos del INIA-Pucallpa para su analizar sus atributos químicos, con énfasis en la concentración de macronutrientes, cuyos resultados son presentados en la Tabla 1.
Posteriormente, los biofertilizantes fueron aplicados 30 días antes de la poda y defoliación, con la finalidad de aportar nutrientes a las plantas y obtener mayor respuesta en las fases de crecimiento vegetativo y productivo. Posteriormente fueron aplicados con una frecuencia de 15 días. En la fase fenológica de floración los biofertilizantes no fueron aplicados con la finalidad de no repeler a los insectos polinizadores. Después de esta etapa la aplicación se realizó normalmente hasta 15 días antes de la cosecha.

El estudio fue instalado en una plantación de siete años de edad establecidas a una densidad de 3 metros entre plantas y 4 metros entre líneas, las plantas presentaron 3,48 m de altura y 2 metros de diámetro de copa en promedio. Las plantas utilizadas para el ensayo procedieron de un estudio de progenies de medios hermanos que fue instalado por el IIAP en el año 2008, con material procedente del banco de germoplasma del INIA Pucallpa. La plantación históricamente viene siendo manejada con limpieza oportuna dentro y fuera de la parcela, así como también con podas de formación, fructificación y sanitaria.

De este modo, el experimento fue conducido mediante un diseño de bloques completamente al azar (DBCA) en esquema factorial $5 \mathrm{~B} \times 5 \mathrm{C}$ con cuatro repeticiones y una planta por unidad experimental. El primer factor (B) estuvo constituido por cinco tipos de biofertilizantes: B1) vacaza (estiércol de vaca), B2) gallinaza (estiércol de gallina de postura), B3) guano de isla (estiércol de aves de mar), B4) pollinaza (estiércol de pollo) y B5) sedimentos de rio, y el segundo factor (C) por cinco concentraciones de biofertilizante: C1) 0, C2) 2, C3) 4, C4) 6 y C5) 8\%, respectivamente. Antes de iniciar el experimento, con la finalidad de uniformizar las etapas fenológicas, las plantas de camucamu fueron sometidas a poda de fructificación y defoliación manual de acuerdo con la metodología de Abanto et al. (2017).

Antes de la aplicación de los tratamientos, cada planta fue dividida en cuatro cuadrantes: Norte, Sur, Este y Oeste. En cada cuadrante fueron seleccionadas dos ramas al azar previamente podadas, luego fueron marcadas con cin-

Tabla 1: Composición química de macronutrientes en los diferentes biofertilizantes después de 90 días de fermentación

\begin{tabular}{lccccc}
\hline \multirow{2}{*}{ Tratamientos } & \multicolumn{5}{c}{ Macronutrientes } \\
\cline { 2 - 6 } & $\mathbf{C a}$ & $\mathbf{M g}$ & $\mathbf{K}$ & $\mathbf{P}$ & $\mathbf{N}$ \\
\cline { 2 - 6 } & & & $\mathbf{\%}$ & 0,13 & 1,00 \\
\hline Sedimentos de Rio (SR) & 2,07 & 0,24 & 0,04 & 1,41 & 1,03 \\
Pollinaza (PO) & 2,91 & 0,16 & 0,06 & 1,46 & 1,02 \\
Gallinaza (GA) & 2,79 & 0,15 & 0,06 & 0,10 & 1,71 \\
Vacaza (VA) & 0,27 & 0,07 & 0,10 & 3,18 & 6,84 \\
Guano de Isla (GI) & 9,65 & 1,43 & 2,76 & \\
\hline
\end{tabular}

Ca, Mg, K, P: Digestión vía seca; Ca, Mg, K: Absorción Atómica; P: Colorimetría; N: Método Micro Keldahl (análisis realizado en INIA, 2015).

Rev. Ceres, Viçosa, v. 66, n.2, p. 108-116, mar/abr, 2019 
tas de colores según la distribución de los tratamientos, en ellas se evaluaron las siguientes características: número de brotes (NB) y número de botones florales (NBF), seguidamente fue evaluado el número de frutos de cosecha (frutos en estado de maduración: $50 \%$ colocarción verde y $50 \%$ coloración rojiza) (NFC), peso de fruto (PF) $(\mathrm{g})$, rendimiento de frutos $(\mathrm{RF})\left(\mathrm{t} \mathrm{ha}^{-1}\right)$ en cada la planta.

Finalmente, las variables analizadas fueron sometidas a análisis de varianza y las medias de los datos cuantitativos fueron analizados a través de regresión polinomial a 5\% de probabilidad. Los análisis estadísticos fueron realizados mediante el software para Sistemas de Análisis de Varianza -SISVAR (Ferreira, 2011).

\section{RESULTADOS Y DISCUSIÓN}

Los resultados del análisis de varianza (ANOVA) expresados en la Tabla 2 muestran el efecto significativo de la interacción entre los tipos y las diferentes concentraciones de biofertilizante según la prueba de $\mathrm{F}(\mathrm{p}>0,05)$ para las variables número de brotes $(\mathrm{NB})$, número de botones florales (NBF), número de frutos de cosecha (NFC), peso de frutos (PF) en gramos por planta y rendimiento de frutos por hectárea $\left(\mathrm{t} \mathrm{ha}^{-1}\right)$.

De manera general fue verificado que las diferentes concentraciones $(\%)$ y tipos de biofertilizantes provocaron tendencia lineal y polinomial cuadrática significativa $(\mathrm{p}>$ 0,05), excepto para número de brotos con los biofertilizantes gallinaza (GA) y sedimentos de rio (SR); de la misma forma el peso de fruto $(\mathrm{PF})$ no fue influenciado significativamente con el biofertilizante sedimentos de rio (SR).

De este modo, el biofertilizante guano de isla (GI) estimuló mayor emisión de brotes que los otros tipos de biofertilizante a medida que se incrementó las concentraciones. En ese sentido, el máximo valor de 1773,73 brotes fue obtenido en respuesta a la concentración de $8 \%$. Del mismo modo, pero en menor cantidad los biofertilizantes vacaza(VA) y pollinaza (PO) obtuvieron 1064,24 y 685 brotes, respectivamente, en la concentración de $8 \%$. Por otra parte, los biofertilizantes gallinaza (GA) y sedimentos de rio (SR) obtuvieron los me- nores resultados de 382 y 151,60 brotes en promedio en todas las concentraciones (Figura 1).

El bajo efecto del biofertilizante sedimentos de rio (SR) sobre la variable, posiblemente fue debido a la poca disponibilidad de nutrientes en relación a los otros biofertilizantes (Tabla 1). En ese sentido se puede afirmar que la baja calidad nutricional del biofertilizante estuvo influenciada por el mayor y menor contenido de arena y arcilla presente en la fuente lo que ocasionó baja retención de nutrientes, dado que el material fue colectado en el rio donde la mayor parte de los nutrientes son transportados por la alta escorrentía.

De otro lado, la mayor emisión de brotes en las plantas de camu-camu tratadas con el biofertilizante guano de isla fue debido a la mayor concentración de $\mathrm{N}$ en la solución, porque según el análisis químico realizado presentó $6,84 \%$ de $\mathrm{N}$, valor superior a las concentraciones de los otros biofertilizantes que presentaron alrededor de 1\% (Tabla 1). Al respecto, Aragão et al. (2011) citan que este efecto ocurre principalmente porque el $\mathrm{N}$ aumenta el crecimiento celular, una vez que este elemento es el principal componente de proteínas y controlador de la absorción de K, P y otros nutrientes en la planta.

Además, la alta eficiencia del $\mathrm{N}$ en la fertilización foliar esta correlacionada directamente con la velocidad de absorción del nutriente, dado que, en cultivos perennes como cítricos, café (Coffea arabica L.) y cacao (Theobroma cacao) varían de 1 a 6 horas para ser absorbido el 50\% del producto aplicado (Malavolta, 2006). Por otro lado, Ciriello et al. (2014) afirman que las plantas, de modo general, responden bien a la fertilización nitrogenada, siendo que el efecto externo del $\mathrm{N}$ más visible es la vegetación verde y abundante, sin embargo, el exceso de $\mathrm{N}$ es perjudicial, por lo que, las dosis aplicadas a los cultivos deben ser equilibradas. Al respecto, Terra (2003) trabajando en el cultivo de plantas de uva (Vitis vinifera L.), verificó que el exceso de $\mathrm{N}$ tornó más vigorosas las plantas, prolongó el periodo de crecimiento vegetativo y retardó la maduración de los frutos.

Tabla 2: Resumen del análisis de varianza para las variables número de brotes (NB), número de botones florales (NBF), número de frutos de cosecha (NFC), peso de fruto (PF) y rendimiento de frutos por hectárea $\left(\mathrm{t} \mathrm{ha}^{-1}\right)$ por efecto de los tipos y diferentes concentraciones (\%) de biofertilizante.

\begin{tabular}{lcccccc}
\hline \multirow{2}{*}{ FV } & GL & \multicolumn{5}{c}{ CM } \\
\cline { 3 - 7 } & & NB & NBF & NFC & PF $(\mathbf{g})$ & R (t ha \\
\hline Bloque & 3 & 280901,9 & 22260,88 & 4445,63 & 0,176 & 0,373 \\
Tipo Biofer (B) & 4 & $4066128,56^{*}$ & $11159082,03^{* *}$ & $5593227,94^{* *}$ & $60,516^{* *}$ & $0,273^{* *}$ \\
Concen. (C) & 4 & $116998,94^{*}$ & $14287017,73^{* *}$ & $4944914,16^{* *}$ & $0,746^{* *}$ & $399,553^{* *}$ \\
B*C & 16 & $211983,20^{*}$ & $3106792,96^{* *}$ & $1252919,96^{* *}$ & $10,130^{* *}$ & $95,000^{* *}$ \\
Residuo & 72 & 45114,43 & 12820,1 & 3604,1 & 15,493 & 454,570 \\
\hline CV $(\%)$ & & 33,06 & 4,86 & 4,8 & 9,09 & 5,12 \\
\hline
\end{tabular}

**, *-Significativo a 1 y $5 \%$ según la prueba de $\mathrm{F}$. 
Para la variable número de botones florales el biofertilizante vacaza (VA) presentó los mejores resultados de 4611,76 en respuesta a la concentración de 6\%. Resultados similares fueron obtenidos por Diniz et al. (2011) trabajando con biofertilizantesa a base de estiércol líquido de bovino en plantas de maracuyá (Passiflora edulis Sims f. flavicarpa Deg). Del mismo modo, pero en menor cantidad los biofertilizantes pollinaza (PO), gallinaza (GA) y sedimentos de rio (SR) obtuvieron 3319,5; 2480,82 y 2215,26 botones florales, respectivamente, en respuesta a la dosis de $5 \%$, luego a partir de esta concentración los valores disminuyeron con el aumento de las dosis de biofertilizante. No en tanto, las concentraciones de biofertilizante guano de isla (GI) provocaron efecto linear creciente, obteniendo un valor máximo de 3306,98 botones florales con la dosis de $8 \%$ (Figura 2).

En ese sentido, es necesario mencionar que el biofertilizante guano de isla (GI), a pesar de poseer la mayor concentración de macronutrientes (Tabla 1) no destacó en esta variable durante el periodo de evaluación dado que el tiempo de floración fue retrasado en vista de la mayor concentración de N. Al respecto, Terra (2003) señala que en esas condiciones provoca el aborto de flores y los brotes y/o ramas tardan en llegar a la madurez fisiológica para la emisión de botones florales. Resultados similares fueron determinados por Abanto et al. (2011), al trabajar con dosis altas de $\mathrm{N}$ en plantas adultas de camu-camu.

Por otro lado, el biofertilizante vacaza (VA) al poseer menor concentración de $\mathrm{N}$ que el biofertilizante guano de isla (GI), pero mayor que los otros biofertilizantes, no tuvo efecto retardante en la etapa fenológica de la emisión de botones florales, por la cual superó a todos los tratamientos.

En relación a la variable número de frutos de cosecha (Figura 3) se observa que los valores máximos fueron registrados en la dosis de $8 \%$ con 3088,85 frutos, en el biofertilizante vacaza (VA). Efectos similares pero en menor cantidad fueron obtenidos por los biofertilizantes pollinaza (PO), gallinaza (GA) y sedimentos de rio (SR), con 1588,$39 ; 1159,36$ y 1142,08 con las dosis $5 ; 4$ y 4\%, respectivamente. Así mismo, el biofertilizante guano de isla (GI) en la concentración de 8\% presentó 2297,56 frutos de cosecha. Resultados inferiores a los verificados con el biofertilizante vacaza (VA) y superiores a los otros biofertilizantes estudiados en este trabajo fueron reportados por Abanto et al. (2015), al trabajar con fertilización foliar a base de biofertilizantes en plantas de camu-camu de 9 años de edad.

De otro lado, la composición nutritiva del biofertilizante vacaza en relación a K también contribuyó en los resultados obtenidos en el presente trabajo (Tabla 1). Visto que, este elemento participa de las actividades metabólicas relativas de síntesis y al transporte de carbohidratos y agua para los frutos. Resultados similares fueron obtenidos por Aular \& Natale (2013), al trabajar con plantas de guayaba (Psidium guajava L.) de la misma familia del camu-camu.

Vale resaltar que, el biofertilizante a base de guano de isla (GI) a pesar de haber presentado la mayor concentración de $2,76 \%$ de $\mathrm{K}$ no tuvo los resultados esperados en relación a número de frutos de cosecha (NFC) y rendimiento de frutos $\left(\mathrm{t} \mathrm{ha}^{-1}\right)$, probablemente debido al atraso en la formación de los botones florales que influencio en la calidad y rendimiento de frutos, y finalmente otro factor muy importante que influyó en los resultados fue el efecto repelente del biofertilizante, porque, según Santos \& Santos (2008) e Vairo dos Santos (1992), el uso constante del biofertilizante reduce el ataque de insectos-plaga, promoviendo un efecto natural de repelencia, principalmente por presentar olores y aromas no atractivos a los insectos. Al respecto, Marcilio et al. (2014) verificaron que el biofertilizante influenció positivamente en el crecimiento

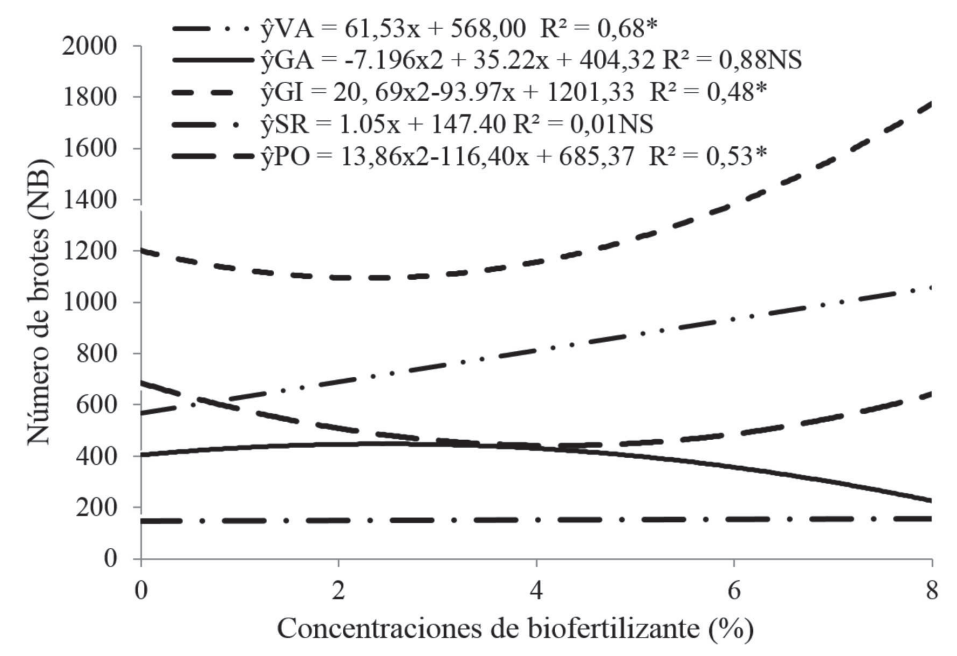

Figura 1: Número de brotes (NB) en plantas de camu-camu por efecto del tipo y diferentes concentraciones de biofertilizante: vacaza (VA), gallinaza (GA), guano de isla (GI), pollinaza (PO) y sedimentos de rio (SR). 
vegetativo y productivo en plantas de banana "Farta velhaco" (Musa spp) y además el biofertilizante utilizado aumentó la resistencia de las plantas al ataque de plagas y patógenos.

El peso de fruto $(\mathrm{g})$ también fue influenciado por las diferentes concentraciones y tipos de biofertilizante (Figura 4), en ese sentido los mejores resultados de $13,76 \mathrm{~g}$ fueron obtenidos por las plantas tratadas con el biofertilizante gallinaza (GA) en respuesta a la dosis de $8 \%$. Por el contrario, los biofertilizantes pollinaza (PO) y guano de isla (GI) tuvieron resultados similares de 10,73 e 10,67 g con la dosis de 5\%, respectivamente. El biofertilizante vacaza (VA) alcanzó un peso de 10,87 g en la dosis de $8 \%$. Además, note se en la Figura 4 que el biofertilizante elaborado de sedimentos de rio (SR) presentó los menores resultados de $6 \mathrm{~g}$ en todas las concentraciones.
En relación al biofertilizante vacaza era de esperarse que también presente los mejores resultados, sin embargo, las plantas al poseer mayor número de frutos presentaran menor peso de fruto, dado que para mantener el número de frutos las plantas no tuvieron las reservas nutritivas suficientes para destinar al incremento del peso del fruto. Resultados similares fueron obtenidos por Abanto et al. (2011) trabajando con dosis altas de N-P y K. Lo contrario fue determinado por Serrano et al. (2007), al evaluar el comportamiento de la poda corta en plantas de guayaba (Psidium guajava L.), en este caso as plantas produjeron menor número frutos, pero de mayor peso.

Por otro lado, según la literatura el peso de fruto varía de acuerdo al tipo de manejo agronómico y al material genético donde se trabaja. De este modo, Abanto et al. (2015) reportaron frutos de 8 a $8,9 \mathrm{~g}$ con biofertilizante de ganado

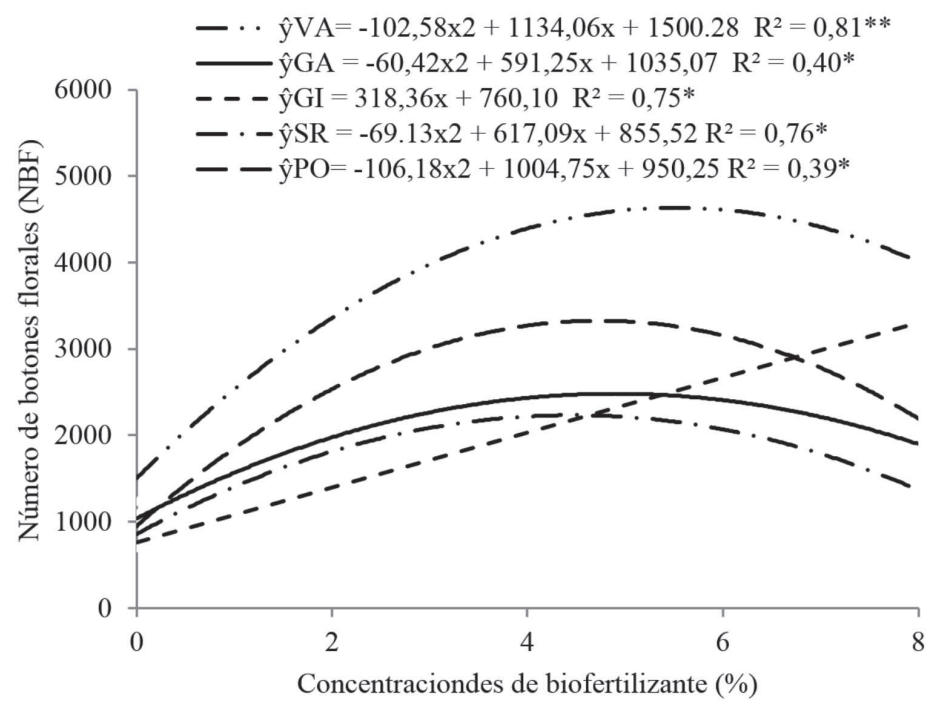

Figura 2: Número de botones florales (NBF) en plantas de camu-camu por efecto del tipo y diferentes concentraciones de biofertilizante: vacaza (VA), gallinaza (GA), guano de isla (GI), pollinaza (PO) y sedimentos de rio (SR).

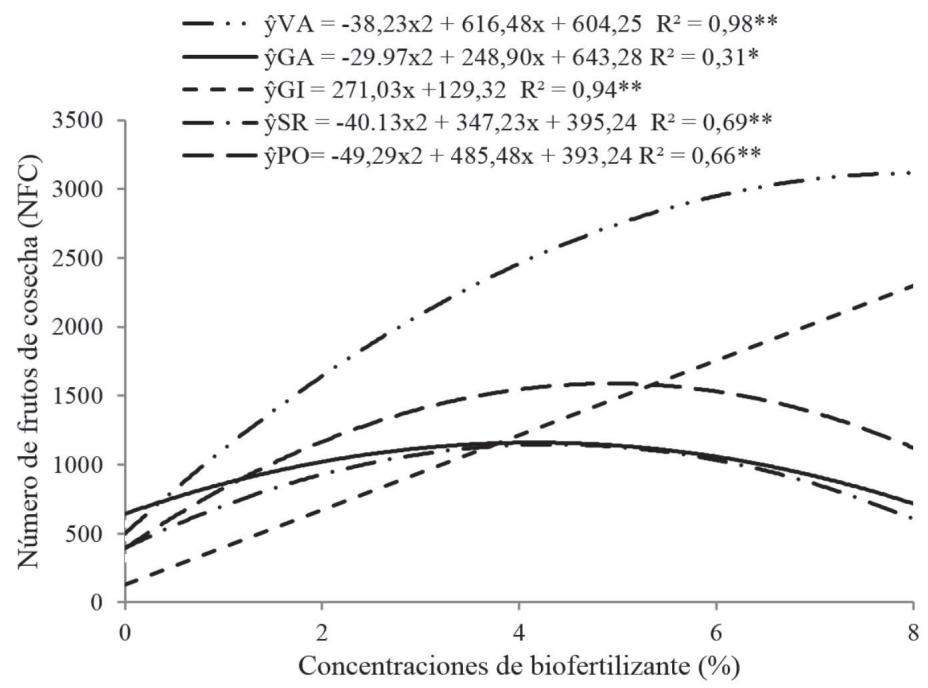

Figura 3: Número de frutos de cosecha (NFC) en plantas de camu-camu por efecto del tipo y diferentes concentraciones de biofertilizante: vacaza (VA), gallinaza (GA), guano de isla (GI), pollinaza (PO) y sedimentos de rio (SR). 
vacuno y ovino. Imán et al. (2011), trabajando con diferentes accesiones promisorias de camu-camu determinaron pesos de frutos entre 8,27 y 15,3 g, siendo la media $11,47 \mathrm{~g}$. Así mismo, Pinedo et al. (2010) evaluaron plantas con 14,91; 13,68 y $12,48 \mathrm{~g}$ en suelos de restinga. Por su parte, Pinto (2011), reportó plantas cuyo peso fue de 24 g trabajando con el uso de materia orgánica, referendo que el factor genético de las plantas se ve favorecido con prácticas de fertilización adecuadas.

El rendimiento de frutos $\left(\mathrm{t} \mathrm{ha}^{-1}\right)$ también fue influenciado por los tratamientos, en ese sentido en la Figura 5 se observa que el biofertilizante vacaza (VA) obtuvo los mejores resultados presentando tendencia lineal creciente en respuesta al aumento de las concentraciones. En ese sentido, la concentración de $8 \%$ obtuvo un valor máximo de 28,87 tha $^{-1}$. Del mismo modo, comportamiento similar, pero con menores resultados fue obtenido por el biofertilizante guano de isla (GI) alcanzando un valor máximo de 19,54 tha ${ }^{-1}$ en respuesta a la concentración de $8 \%$. De otro lado, el biofertilizante pollinaza (PO), gallinaza (GA) y sedimentos de rio (SR) presentaron ajuste cuadrático significativo, obteniendo como valor máximo 13,8; 11,25 y 6,17 t ha $^{-1}$, respectivamente, en respuesta a la concentración de $5 \%$.

Resultados inferiores fueron determinados por Abanto et al. (2015), en plantas de camu-camu de 9 años de edad en suelos de restinga. Los biofertilizantes elaborados a base de estiércol de ganado ovino y vacuno provocaron aumentos significativos en la producción, obteniendo 17,53 y 14,18 tha $^{-1}$, respectivamente. Notese, que estos resultados están por debajo de los encontrados con los biofertilizantes vacaza (VA) y guano de isla (GI), pero fueron superiores a los otros biofertilizantes. De igual modo, Pérez (2009) trabajando con fertilización foliar

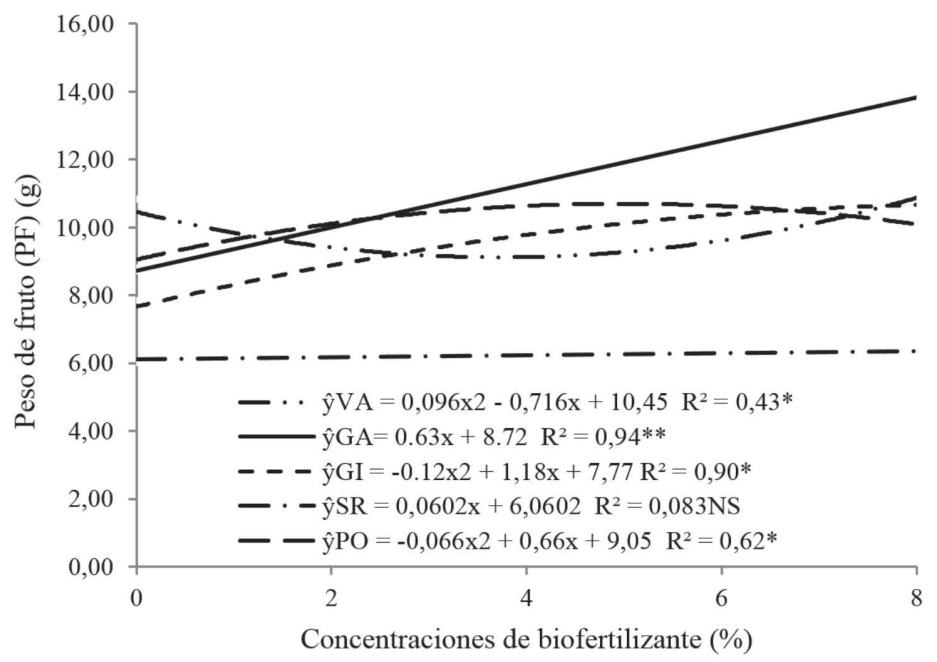

Figura 4: Peso de frutos (PF) (g) en plantas de camu-camu por efecto del tipo y diferentes concentraciones de biofertilizante: vacaza (VA), gallinaza (GA), guano de isla (GI), pollinaza (PO) y sedimentos de rio (SR).

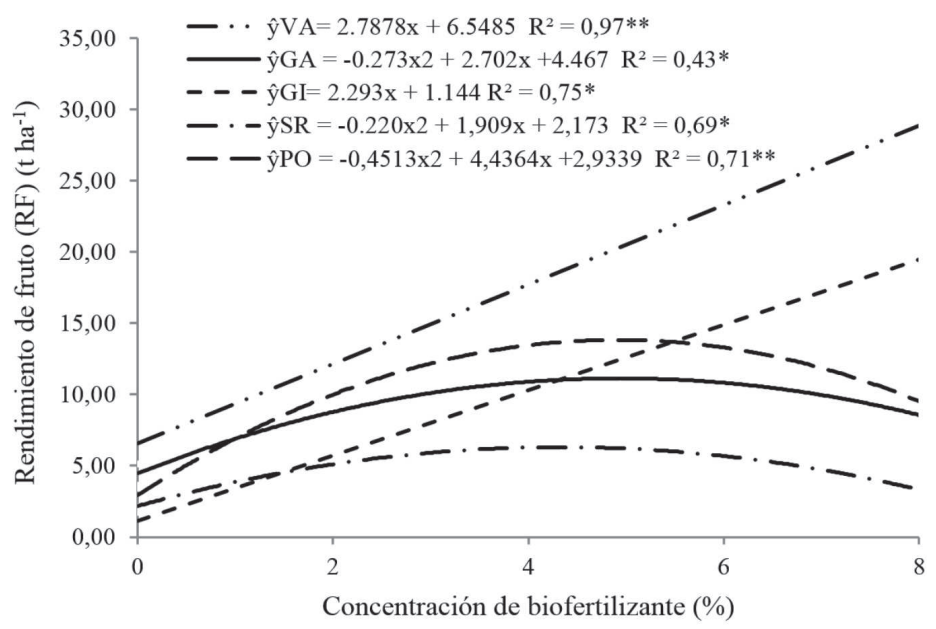

Figura 5: Rendimiento de frutos (RF) $\left(\mathrm{t} \mathrm{ha}^{-1}\right)$ en plantas de camu-camu por efecto del tipo y diferentes concentraciones de biofertilizante: vacaza (VA), gallinaza (GA), guano de isla (GI), pollinaza (PO) y sedimentos de rio (SR). 
orgánica a base de biofertilizantes en plantas de camucamu de 9 años de edad determinaron un rendimiento de $15,4 \mathrm{t} \mathrm{ha}^{-1}$ de frutos con biofertilizante ovinaza en restinga baja inundable.

En lo que respecta a este estudio, la correcta aplicación y calidad nutricional de cada uno de los biofertilizantes, acompañado con manejo de podas de fructificación, defoliación, eliminación de ritidoma de los tallos principales y manejo oportuno de plantas dañinas dentro y alredores de la parcela realizados por el produtor permitió obtener resultados satisfactorios.

Así también se destaca que durante la conducción del experimento no fue aplicado productos para el control de plagas. En ese sentido, los resultados positivos son atribuidos al efecto repelente del biofertilizante, coincidiendo con lo afirmado con Vairo dos Santos et al. (1992).

\section{CONCLUSIONES}

El biofertilizante elaborado de estiércol de vaca, incrementó la calidad y el rendimiento de frutos de camucamu en suelos de restinga.

El biofertilizante elaborado con sedimentos de rio no proporcionó efectos positivos sobre las caracteristicas vegetativas y productivas de las plantas de camu-camu.

El biofertilizante guano de isla (GI) fue el segundo en presentar los mejores resultados, sin embargo, el alto contenido de $\mathrm{N}$ retardó o desarrollo normal de las fases fenológicas vegetativas y productivas, de este modo se recomienda realizar más estudios de tiempo de evaluación y de diferentes concentraciones para determinar su máxima efectividad.

\section{AGRADECIMIENTOS}

Los autores agradecen al Programa Nacional de Innovación para la Competitividad y Productividad (INNOVATE-PERU) por el financiamiento para realizar el presente trabajo de investigación en el marco del Convenio: 403-PNICT-PIAP-2014: Sistema de producción orgánica del camu-camu Myrciaria dubia en humedales de Loreto y Ucayali, y al Sr. Adolfo Bardales por dar las facilidades para realizar este estudio en su plantación de camu-camu.

\section{REFERENCIAS}

Abanto RC, Oliva C, Domínguez G, Meza A \& Alves EC (2011) Fertirriego en la producción del camu-camu (Myrciaria dubia HBK Mc Vaugh) en la estación experimental del IIAP, Ucayali, Perú. Scientia Agropecuaria, 02:157-167.

Abanto RC, Chagas EA, Sánchez-Choy J, dos Santos VA, Bardales LR \& Saldaña RG (2014) Capacidad de enraizamiento de plantas matrices promisorias de Myrciaria dubia (Kunth) Mc Vaugh en cámaras de subirrigación. Revista Ceres, 06:134-140.
Abanto RC, Del Castillo DT, Chagas EA \& Sakazaki RT (2015) Efecto de la fertilización orgánica en la producción y calidad de frutos de plantas de camu camu en Ucayali-Perú. In: IX Congresso Brasileiro de Agroecologia, Belém. Anais, CBA 3 / Cadernos de Agroecologia. p. 01-06.

Abanto-Rodriguez C, Pinedo-Panduro M, Alves-Chagas E, Cardoso-Chagas P, Tadashi-Sakazaki R, Santos de Menezes PH, Farias-Araújo W \& Murga-Orrillo H (2016) Relation between the mineral nutrients and the Vitamin $\mathrm{C}$ content in camu-camu plants (Myrciria dubia) cultivated on high soils and flood soils of Ucayali, Peru. Scientia Agropecuaria, 07:297-304.

Abanto RC, Chagas EA, Chagas PC, del Castillo TD, Sakazaki RT \& Delgado MJP (2017) Effects of different times and intensities of fructification pruning in camu-camu plants. Fruits, 72:109118 .

Aragão VF, Fernandes PD, Gomes Filho RR, Santos Neto AM, Carvalho CM \& Feitosa HO (2011) Efeito de diferentes lâminas de irrigação e níveis de nitrogênio na fase vegetativa do pimentão em ambiente protegido. Revista Brasileira de Agricultura Irrigada, 05:361-375.

Aular J \& Natale W (2013) Nutrição mineral e qualidade do fruto de algumas frutíferas tropicais: goiabeira, mangueira, bananeira e mamoeiro. Revista Brasileira Fruticultura, 35:1214-1231.

Alves RE, Filgueiras HAC, Moura CFH, Araujo NCC \& Almeida AS (2002) Camu-camu (Myrciaria dubia Mc Vaugh): A rich natural source of vitamin C. Proceedings of the InterAmerican Society for Tropical Horticulture, 46:11-13.

Chatterjee IB, Majumber AK, Nandi BK \& Subramanian N (1975) Synthesis and some major functions of vitamin $\mathrm{C}$ in animals. Ann. N.Y. Academy of Sciences, 258:24-47.

Ciriello V, Guerrini IA \& Backes C (2014) Doses de nitrogênio no crescimento inicial e nutrição de plantas de guanandi. CERNE, 20:653-660.

Diniz AA, Cavalcante LF, Rebequi AM, Nunes JC \& Brehm MAS (2011) Esterco líquido bovino e uréia no crescimento e produção de biomassa do maracujazeiro amarelo. Revista Ciência Agronômica, 42:597-604.

Dirección Regional Agraria de Loreto (DRAL) (2015) Informe de agencias agrarias. Oferta comercial de camu camu, en la Región Loreto. 6p.

Dirección Regional Sectorial de Agricultura Ucayali (DRSAU) (2017) Informe situacional de la cadena productiva de camucamu, Dirección Regional Sectorial de Agricultura. Pucallpa, Perú. 16p.

Ferreira DF (2011) Sisvar: a computer statistical analysis system. Ciência e Agrotecnologia, 35:1039-1042.

Imán CS, Pinedo FS \& Melchor AM (2011) Caracterización morfológica y evaluación de la colección nacional de germoplasma de camu camu Myrciaria dubia (H.B.K) Mc Vaugh, del INIA Loreto-Perú. Scientia Agropecuaria, 02:189-201.

Marcilio HC, Ramos MJM, de Andrade AL, da Silva EC \& dos Santos CC (2014) Efeito de biofertilizante no crescimento e na produção da bananeira 'farta velhaco', no sudoeste de Mato Grosso. Revista Brasileira de Agropecuária Sustentável (RBAS), 04:131-135.

Medeiros MB \& Lopes JS (2006) Biofertilizantes líquidos e sustentabilidade agrícola. Bahia Agrícola, 07:24-26.

Malavolta E (2006) Absorção, transporte e redistribuição. In: Malavolta E (Ed.) Manual de nutrição mineral de plantas. São Paulo, Editora Agronômica Ceres Ltda. p.57-112. 
Neves LC, Silva VX, Pontis JA, Flach A \& Roberto SR (2015) Bioactive compounds and antioxidant activity in preharvestcamu-camu [Myrciaria dubia (H.B.K.) Mc Vaugh] fruits. Scientia Horticulturae, 186:223-229.

Naidu KA (2003) Vitamin C in human health and disease is still a mystery? An overview. Journal of Nutrition, 02:07-23.

Pérez EAM (2009) Efecto de la fertilización foliar orgánica a base de bioles en la producción de camu camu (Myrciaria dubia H.B.K Mc Vaugh) en un entisols de Pucallpa. Tesis para optar el título de Ingeniero. Universidad Nacional de Ucayali, Pucallpa. $81 \mathrm{p}$

Pinedo PM, Delgado VC, Farroñay PR, Imán CS, Villacrés VJ, Faching ML, Oliva CC, Abanto RC, Bardales LR \& Vega VR (2010) Camu-Camu (Myrciaria dubia- Myrtaceae): Aportes para su Aprovechamiento Sostenible en la Amazonia Peruana Iquitos, FINCyT. 130p.

Pinto LCED (2011) Produção e qualidade do fruto de camu-camu sob efeito de adubação orgânica e mineral no município de Presidente Figueiredo-AM. Dissertação de mestrado. Instituto Nacional de Pesquisas da Amazônia, Manaus. 48p.

Santos dos JGR \& Santos dos ECXR (2008) Agricultura orgânica: teoria e prática. Campina Grande, Editora da Universidade Estadual da Paraíba-EDUEP. 230p.
Serrano LAL, Marinho CS, Ronchi CP, Lima IM, Martins MVV \& Tardin FD (2007) Goiabeira 'Paluma' sob diferentes sistemas de cultivo, épocas e intensidades de poda de frutificação. Pesquisa Agropecuária Brasileira, 42:785-792.

SUNAT-Superintendencia Nacional de Aduanas y de Administración Tributaria (2018) Exportaciones del producto camu-camu según sus principales presentaciones y mercados, 2013-2018. Disponible en:<http://www.siicex.gob.pe/siicex/apb/ReporteProducto aspx ?psector $=1025 \&$ preporte $=$ prodpresvolu $\&$ pvalor $=$ 1920>. Acesado en: 10 de abril de 2018.

Souza BR \& Alcântara AF (2007) Adubação Orgânica. In: Gilmar Paulo Henz, Flávia Aparecida de Alcântara \& Francisco Vilela Resende (Eds.) Produção orgânica de hortaliças: o produtor pergunta, a Embrapa responde. Brasília, Dstrito Federal. p.113127.

Terra MM (2003) Nutrição, calagem e adubação. In: Pommer CV (Ed.) Uva: Tecnologia de produção, pós-colheita, mercado. Rio Grande do Sul, Porto Alegre. p.405-476.

Vairo dos Santos AC (1992) Biofertilizante líquido: o defensivo agrícola da natureza. $2^{\mathrm{a}}$ ed. Niterói, EMATER-RJ. 16p. 